\title{
An all-particle primary energy spectrum in the 3-200 PeV energy range
}

\author{
A P Garyaka ${ }^{1}$, R M Martirosov ${ }^{1}$, S V Ter-Antonyan ${ }^{2,7}$, A D Erlykin ${ }^{3}$, \\ N M Nikolskaya ${ }^{3}$, Y A Gallant ${ }^{4}$, L W Jones ${ }^{5}$ and J Procureur ${ }^{6}$ \\ 1 Yerevan Physics Institute, 2 Alikhanyan Br. Str., 375036 Yerevan, Armenia \\ 2 Department of Physics, Southern University, Baton Rouge, USA \\ ${ }^{3}$ P N Lebedev Physical Institute, Moscow, Russia \\ ${ }^{4}$ LPTA, Université Montpellier II, CNRS/IN2P3, Montpellier, France \\ ${ }^{5}$ Department of Physics, University of Michigan, USA \\ ${ }^{6}$ Centre d'Etudes Nucléaires de Bordeaux-Gradignan, Gradignan, France \\ E-mail: samvel_terantonyan@subr.edu
}

Received 2 April 2008

Published 12 August 2008

Online at stacks.iop.org/JPhysG/35/115201

\begin{abstract}
We present an all-particle primary cosmic-ray energy spectrum in the $3 \times 10^{6}$ $2 \times 10^{8} \mathrm{GeV}$ energy range obtained by a multi-parametric event-by-event evaluation of the primary energy. The results are obtained on the basis of an expanded EAS data set detected at mountain level $\left(700 \mathrm{~g} \mathrm{~cm}^{-2}\right)$ by the GAMMA experiment. The energy evaluation method has been developed using the EAS simulation with the SIBYLL interaction model taking into account the response of GAMMA detectors and reconstruction uncertainties of EAS parameters. Nearly unbiased $(<5 \%)$ energy estimations regardless of a primary nuclear mass with an accuracy of about $15-10 \%$ in the $3 \times 10^{6}-2 \times$ $10^{8} \mathrm{GeV}$ energy range respectively are attained. An irregularity ('bump') in the spectrum is observed at primary energies of $\sim 7.4 \times 10^{7} \mathrm{GeV}$. This bump exceeds a smooth power-law fit to the data by about 4 standard deviations. By not rejecting the stochastic nature of the bump completely, we examined the systematic uncertainties of our methods and conclude that they cannot be responsible for the observed feature.
\end{abstract}

\section{Introduction}

Study of the fine structure in the primary energy spectrum is one of the most important tasks in the very high energy cosmic ray experiments [1]. Commonly accepted values of the allparticle energy spectrum indices of -2.7 and -3.1 before and after the knee are an average and do not reflect the real behavior of the spectrum particularly after the knee. It is necessary

7 Author to whom any correspondence should be addressed. 


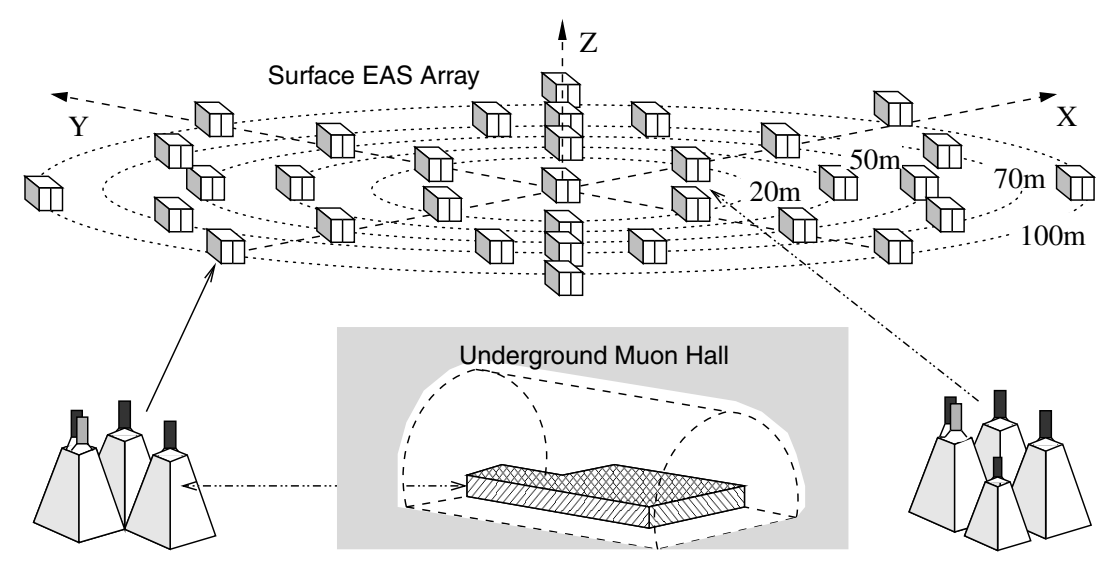

Figure 1. Diagrammatic layout of the GAMMA facility.

to pay special attention to the energy region of $(1-10) \times 10^{7} \mathrm{GeV}$, where experimental results have been very limited up to now. Irregularities of the energy spectrum in this region were observed a long time ago. They can be seen from the energy spectrum obtained more than 20 years ago in the AKENO experiment [2] as well as in later works of the GAMMA [3] and TUNKA [4] experiments. At the same time the large statistical errors did not allow us to discuss the reasons for these irregularities.

On the other hand results of many experiments on the study of EAS charge particle spectra, and the behavior of the age parameter and muon component characteristics point out that the primary mass composition behind the knee becomes significantly heavier. Based on these indications, additional investigations into the fine structure of the primary energy spectrum at $(1-10) \times 10^{7} \mathrm{GeV}$ have an obvious interest.

There are two ways of obtaining the primary energy spectra using detected extensive air showers (EAS). The first way is a statistical method which unfolds the primary energy spectra from the corresponding integral equation set based on a detected EAS data set and the model of the EAS development in the atmosphere [5-9]. The second method is based on an eventby-event evaluation [2, 10-13] of the primary energy of the detected EAS with parameters $\mathbf{q} \equiv q\left(N_{\mathrm{e}}, N_{\mu}, N_{\mathrm{h}}, s, \theta\right)$ using parametric $E=f(\mathbf{q})[2,10,11,13]$ or non-parametric [12] energy estimator previously determined on the basis of shower simulations in the framework of a given model of EAS development.

Here, applying a new event-by-event parametric energy evaluation $E=f(\mathbf{q})$, the allparticle energy spectrum in the knee region is obtained on the basis of the data set obtained using the GAMMA EAS array $[7-9,11]$ and a simulated EAS database obtained using the SIBYLL [14] interaction model. Preliminary results have been presented in $[10,11]$.

\section{GAMMA experiment}

The GAMMA installation [7-9, 11] is a ground-based array of 33 surface detection stations and 150 underground muon detectors, located at the south side of Mount Aragats in Armenia. The elevation of the GAMMA facility is $3200 \mathrm{~m}$ above sea level, which corresponds to $700 \mathrm{~g} \mathrm{~cm}^{-2}$ of atmospheric depth. A diagrammatic layout of the array is shown in figure 1 . 
The surface stations of the EAS array are arranged in five concentric circles of $\sim 20,28$, 50,70 and $100 \mathrm{~m}$ radii, and each station contains plastic scintillation detectors with dimensions of $1 \times 1 \times 0.05 \mathrm{~m}^{3}$. Each of the central nine stations contains an additional (the 4th) small scintillator with dimensions of $0.3 \times 0.3 \times 0.05 \mathrm{~m}^{3}$ (figure 1 ) for high particle density $\left(\gg 10^{2}\right.$ particles $\left.\mathrm{m}^{-2}\right)$ measurements.

A photomultiplier tube is placed on the top of the aluminum casing covering each scintillator. One of the three detectors of each station is viewed by two photomultipliers, one of which is designed for fast timing measurements.

One hundred and fifty underground muon detectors ('muon carpet') are compactly arranged in the underground hall under $2.3 \mathrm{~kg} \mathrm{~cm}^{-2}$ of concrete and rock. The scintillator dimensions, casings and photomultipliers are the same as in the EAS surface detectors.

The shower size thresholds of the $100 \%$ shower detection efficiency are equal to $N_{\text {ch }}=3 \times 10^{5}$ and $N_{\text {ch }}=5 \times 10^{5}$ at the EAS core location within $R<25 \mathrm{~m}$ and $R<50 \mathrm{~m}$ respectively $[7,8,11]$.

The time delay is estimated by the pair-delay method [15] to give the time resolution of about 4-5 ns. The EAS detection efficiency $\left(P_{d}\right)$ and the corresponding shower parameter reconstruction errors are equal to: $P_{d}=100 \%, \Delta \theta \simeq 1.5^{\circ}, \Delta N_{\mathrm{ch}} / N_{\mathrm{ch}} \simeq 0.05-$ $0.15, \Delta s \simeq 0.05, \Delta x$ and $\Delta y \simeq 0.7-1 \mathrm{~m}$. The reconstruction errors of the truncated muon shower sizes for $R_{\mu}<50 \mathrm{~m}$ from the shower core are equal to $\Delta N_{\mu} / N_{\mu} \simeq 0.2-0.35$ at $N_{\mu} \simeq 10^{5}-10^{3}$ respectively $[8,9,11]$.

\section{Event-by-event energy estimation}

\subsection{Key assumptions}

Suppose that $E_{1}=f(\mathbf{q})$ is an estimator of energy $E_{0}$ of unknown primary nuclei which induced showers with the detected parameter $\mathbf{q} \equiv q\left(N_{\mathrm{ch}}, N_{\mu}, s, \theta\right)$. Then the expected allparticle energy spectrum $F\left(E_{1}\right)$ is defined by

$$
F\left(E_{1}\right)=\int \Im\left(E_{0}\right) W\left(E_{0}, E_{1}\right) \mathrm{E}_{0},
$$

where $\Im\left(E_{0}\right)$ is the energy spectrum of primary nuclei and $W\left(E_{0}, E_{1}\right)$ is the corresponding $\left(E_{0}, E_{1}\right)$ transformation probability density function.

If $\Im\left(E_{0}\right) \propto E_{0}^{-\gamma}$ and $W\left(E_{0}, E_{1}\right)$ are the log-normal distributions with $\delta=\overline{E_{1} / E_{0}}$ and $\sigma$ parameters, expression (1) has analytic solution for the expected spectrum of the energy estimator [16]:

$$
F\left(E_{1}\right)=\Im\left(E_{0}\right) \delta^{\gamma-1} \exp \left(\frac{((\gamma-1) \sigma)^{2}}{2}\right) .
$$

It is seen that evaluation of energy spectrum $\Im\left(E_{0}\right)$ from (2) is possible to perform only at a priori known $\gamma, \delta$ and $\sigma$ parameters and spectral slope $(\gamma)$ of detected energy spectra $F\left(E_{1}\right)$ coincides with spectral slope of primary energy spectra $\Im\left(E_{0}\right)$. The values of $\delta$ and $\sigma$ may depend on the primary energy $\left(E_{0}\right)$ and mass of primary nuclei $(A)$ that the all-particle energy spectrum $\Im\left(E_{0}\right)=\sum_{A} \mathfrak{\Im}_{A}\left(E_{0}\right)$ is consisted of. In this case, expression (1) is unfolded numerically and the slope of the detected energy spectrum can differ from the primary energy spectrum.

For example, the dependence $\sigma\left(E_{0}\right)=a \ln \left(E_{0} / E_{0, \min }\right)+b$ at $|a|<0.1$ leads to the numerical solutions which can be approximated by expression (2) replacing $\sigma$ with $\sigma\left(E_{0}\right)-a \sqrt{\gamma}$. The corresponding approximation errors are about 2-5\% in the energy range of $E_{\min }-500 E_{\min }$ and $\gamma \simeq 2.3-3.2$. 
However, the evaluation of energy spectra can be simplified provided

$$
\begin{aligned}
& \gamma\left(E_{0}\right) \simeq \gamma \pm \Delta \gamma \\
& \delta\left(E_{0}\right) \simeq \delta_{A}\left(E_{1}\right) \simeq \delta \equiv 1 \pm \Delta \delta\left(E_{1}\right) \\
& \sigma\left(E_{0}\right) \simeq \sigma \pm \Delta \sigma
\end{aligned}
$$

are satisfied for a given energy range of $E_{1}$. Then, the all-particle energy spectrum can be evaluated from

$$
\Im\left(E_{0}\right)=F\left(E_{1}\right) \exp \left(-\frac{((\gamma-1) \sigma)^{2}}{2}\right) .
$$

The corresponding error of evaluation (6) with approaches (3)-(5) is determined by a sum of the statistical errors $\Delta F\left(E_{1}\right)$ and systematic errors $\eta$ due to approaches being used:

$$
\left(\frac{\Delta \mathfrak{I}}{\mathfrak{I}}\right)^{2} \simeq\left(\frac{\Delta F}{F}\right)^{2}+\eta^{2}
$$

where the systematic relative errors $\eta$ are

$$
\eta^{2} \simeq(\Delta \delta(\gamma-1))^{2}+\left[(\sigma(\gamma-1))^{2}\left(\frac{\Delta \sigma}{\sigma}+\frac{\Delta \gamma}{\gamma-1}\right)\right]^{2} .
$$

The values of $\gamma, \delta$ and $\sigma$ parameters from expressions (3)-(7) and the corresponding uncertainties $\Delta \gamma, \Delta \delta$ and $\Delta \sigma$ essential for the reconstruction of primary energy spectrum using the GAMMA facility EAS data and approach (6) are considered in sections 3.2-3.4 .

\subsection{Uncertainty of a spectral slope}

The results of different experiments [9, 17-19] and theoretical predictions [20-22] indicate that the elemental energy spectra can be presented in the power-law form

$$
\Im_{A}(E) \propto\left(\frac{E}{E_{k}}\right)^{-\gamma},
$$

where $\gamma=\gamma_{1} \simeq 2.7_{-0.1}^{+0.05}$ at $E<E_{k}(A)$ and $\gamma=\gamma_{2} \simeq 3.15_{-0.05}^{+0.1}$ at $E>E_{k}(A)$. It is also accepted that the mass spectra of primary nuclei can be divided into separate nuclear groups and below, as in [9], just four nuclear species (H, He, O-like and Fe-like) are considered. Dependence of the knee energy $E_{k}$ on the primary nuclei type is assumed to be either rigidity dependent, $E_{k}=Z E_{Z=1}[9,20-22]$ or $A$-dependent $[9,23], E_{k}=A E_{A=1}$, where $Z$ and $A$ are the charge and mass of primary nuclei, respectively.

As a result, the all-particle energy spectrum $\sum_{A} \mathfrak{\Im}_{A}(E)$ slowly changes its slope and can be roughly approximated by a power-law spectrum with power index $\gamma \simeq 2.7$ at $E<3 \times$ $10^{6} \mathrm{GeV}, \gamma \simeq 2.9$ at $3 \times 10^{6}<E<10^{7} \mathrm{GeV}$ and $\gamma \simeq 3.1$ at $E>10^{7} \mathrm{GeV}$. This presentation of the all-particle spectrum agrees with world data [23] in the $\Delta \gamma \simeq 0.1$ range of uncertainty and energy interval $10^{6}<E<2 \times 10^{8} \mathrm{GeV}$.

The values of $\Delta \delta_{A}(E)$ and $\sigma_{A}\left(E_{0}\right)$ parameters are presented in section 3.4 and depend on efficiency of energy estimator $E_{1}=f\left(N_{\mathrm{ch}}, N_{\mu}, s, \theta\right)$.

Note that it follows from expression (7) that for $\sigma \simeq 0.1-0.15$ and $\Delta \sigma=0.03$ the contribution of $\Delta \gamma$ to the systematic errors (7) is negligible and the difference of all-particle spectra evaluated by expression (6) for $\gamma=2.7$ and $\gamma=3.1$ is less than $2 \%$ at $\sigma \simeq 0.15$. 


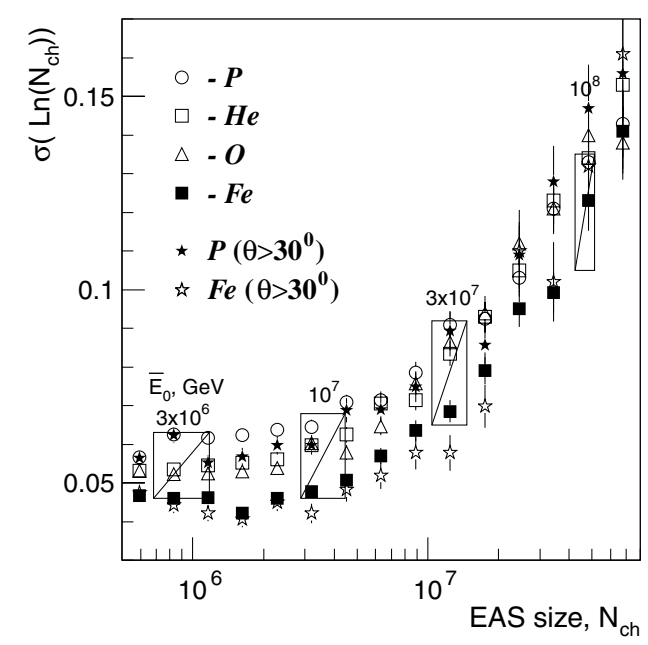

Figure 2. Shower size reconstruction errors for different primary nuclei (p, He, O, Fe) and zenith angles $\left(\theta<45^{\circ}\right.$ and $\left.30^{\circ}<\theta<45^{\circ}\right)$. The right and left ends of the diagonals of the rectangle show the average primary energies $\left(\overline{E_{0}}\right)$ and the corresponding shower sizes computed for the primary proton and iron nuclei, respectively.

\subsection{The simulated EAS database}

To obtain the parametric representation for the unbiased $(\delta \simeq 1)$ energy estimator $E_{1}$ of the primary energy $E_{0}$ we simulated showers database using the CORSIKA(NKG) EAS simulation code [24] with the SIBYLL [14] interaction model for $\mathrm{H}, \mathrm{He}, \mathrm{O}$ and Fe primary nuclei.

Preliminary, the showers simulated with NKG mode of CORSIKA code for each of the primary nuclei compared with the corresponding simulations using EGS mode of CORSIKA [24] taking into account the detector response, contribution of EAS $\gamma$-quanta and shower parameter reconstruction uncertainties. Simulated statistics were equal to 200 events for each of primary nuclei with log-uniform primary energy distribution in the range of $2 \times 10^{6}$ $10^{8} \mathrm{GeV}$. Using the threshold energy of shower electrons (positrons) for NKG mode at observation level as a free parameter (the same as it was performed in [9]), the biases $\left.\delta\left(N_{\mathrm{ch}}, A\right)=\left(N_{\mathrm{ch}}(\mathrm{NKG}) / N_{\mathrm{ch}}(\mathrm{EGS})\right)-1\right)$ and $\delta_{s}(A)=s(\mathrm{NKG})-s(\mathrm{EGS})$ were minimized for all the simulated primary nuclei $(\mathrm{H}, \mathrm{He}, \mathrm{O}, \mathrm{Fe})$.

The applied method of calibration of the NKG mode of CORSIKA for the GAMMA EAS array differed from [9] only by the expanded range of selected shower core coordinates $(R<50 \mathrm{~m})$ and zenith angles $\theta<45^{\circ}$. The obtained biases of shower size $\delta\left(N_{\mathrm{ch}}\right)$ and age parameter $s$ in the range of statistical errors $(<5 \%)$ agreed with data [9]. The values of $\delta\left(N_{\mathrm{ch}}\right)$ were used further for the correction of the shower size obtained using the NKG simulation mode.

The simulated primary energies $\left(E_{0}\right)$ for the shower database were distributed according to a power-law spectrum $I\left(E_{0}\right) \propto E_{0}^{-1.5}$ with $\mathcal{N}=2 \times 10^{4}$, total number of detected $\left(N_{\mathrm{ch}}>5 \times 10^{5}, R<50 \mathrm{~m}\right)$ and reconstructed showers for each primary nucleus. The energy thresholds of primary nuclei were set as $E_{0, \min }(A) \equiv 10^{6} \mathrm{GeV}$ and $E_{\max }=5 \times 10^{8} \mathrm{GeV}$. The simulated showers had core coordinates distributed uniformly within the radius of $R<75 \mathrm{~m}$ and zenith angles $\theta<45^{\circ}$.

The reconstruction errors $\sigma\left(\ln N_{\mathrm{ch}}\right)$ of shower size $N_{\mathrm{ch}}$ are presented in figure 2 for different primary nuclei and different zenith angles. The right and left ends of the diagonals 
Table 1. Correlation coefficients $\rho\left(\mathbf{q}, \ln E_{0}\right)$ and $\rho(\mathbf{q}, \ln A)$ between shower parameter $\mathbf{q} \equiv$ $q\left(N_{\mathrm{ch}}, N_{\mu}, s\right)$ and primary energy (ln $\left.E_{0}\right)$ and nuclei mass $\ln A$ for two zenith angular intervals.

\begin{tabular}{lcccc}
\hline $\mathbf{q}$ & $\ln E_{0},\left(\theta<10^{\circ}\right)$ & $\ln E_{0},\left(\theta<45^{\circ}\right)$ & $\ln A,\left(\theta<10^{\circ}\right)$ & $\ln A,\left(\theta<45^{\circ}\right)$ \\
\hline $\ln N_{\text {ch }}$ & $0.986 \pm 0.001$ & $0.954 \pm 0.0004$ & $0.013 \pm 0.013$ & $0.007 \pm 0.004$ \\
$\ln N_{\mu}$ & $0.978 \pm 0.001$ & $0.969 \pm 0.0003$ & $0.139 \pm 0.012$ & $0.132 \pm 0.004$ \\
$s$ & $-0.029 \pm 0.013$ & $-0.02 \pm 0.004$ & $0.018 \pm 0.013$ & $0.015 \pm 0.004$ \\
\hline
\end{tabular}

of the rectangle in figure 2 show the average primary energies (in units of $\mathrm{GeV}$ ) responsible for corresponding shower sizes for the primary proton and Iron nuclei respectively.

All EAS muons with energies of $E_{\mu}>4 \mathrm{GeV}$ at the GAMMA observation level have passed through the $2.3 \mathrm{~kg} \mathrm{~cm}^{-2}$ of rock to the muon scintillation carpet (the underground muon hall, figure 1). The muon ionization losses and electron (positron) accompaniment due to muon electromagnetic and photonuclear interactions in the rock are taken into account using the approximation for equilibrium accompanying charged particles obtained from preliminary simulations with the FLUKA code [26] in the $0.005-20 \mathrm{TeV}$ muon energy range. The resulting charged particle accompaniment per EAS muon in the underground hall is equal to $0.06 \pm 0.01(100 \% \mathrm{e})$ and $11.0 \pm 1.5(98.5 \% \mathrm{e}, 1.4 \% \mathrm{~h}, 0.04 \% \mu)$ at muon energies $0.01 \mathrm{TeV}$ and $10 \mathrm{TeV}$ respectively.

Due to the absence of saturation in the muon scintillation carpet, the reconstruction errors $\left(\Delta \ln N_{\mu}\right.$ ) of the truncated muon size $N_{\mu}$ continuously decrease with increasing muon truncated sizes in the range $10^{3}<N_{\mu}<10^{5}$. Corresponding magnitudes of reconstruction errors for primary protons and Iron nuclei were equal to $\Delta\left(\ln N_{\mu, \mathrm{p}}\right) \simeq 0.35,0.18,0.15$ and $\Delta\left(\ln N_{\mu, \mathrm{Fe}}\right) \simeq 0.38,0.19,0.10$ for EAS muon truncated sizes $N_{\mu} \simeq 10^{3}, 10^{4}, 10^{5}$ respectively.

Fluctuations of the shower size for given primary energies $E_{0, A} \equiv 10^{6}, 10^{7}, 10^{8} \mathrm{GeV}$ and $\cos \theta<0.95$ were equal to $\sigma_{A \equiv \mathrm{p}}\left(N_{\mathrm{ch}}, E_{0}\right) \simeq 0.20,0.14,0.10$ and $\sigma_{A \equiv \mathrm{Fe}}\left(N_{\mathrm{ch}}, E_{0}\right) \simeq$ $0.16,0.13,0.08$, respectively. The corresponding fluctuations of muon truncated size were equal to $\sigma_{A \equiv \mathrm{p}}\left(N_{\mu}, E_{0}\right) \simeq 0.25,0.23,0.2$ and $\sigma_{A \equiv \mathrm{Fe}}\left(N_{\mu}, E_{0}\right) \simeq 0.13,0.10,0.08$. For zenith angles of primary nuclei $45^{\circ}>\theta>30^{\circ}$, the fluctuations increase by about 1.5-2 times due to the aging of detected showers.

The $4 \times 2 \times 10^{4}$ EAS simulated events with reconstructed $N_{\mathrm{ch}}, N_{\mu}(R<50 \mathrm{~m}), s$ and $\theta$ shower parameters for the $E_{0}$ and $A$ parameters of primary nuclei made up the simulated EAS database.

\subsection{Energy estimator}

The event-by-event reconstruction of the primary all-particle energy spectrum using the GAMMA facility is mainly based on high correlation of primary energy $E_{0}$ and shower size $\left(N_{\mathrm{ch}}\right)$. The shower age parameter $(s)$ zenith angle $(\theta)$ and muon truncated shower size $\left(N_{\mu}\right)$ have to decrease the unavoidable biases of energy evaluations due to abundance of different primary nuclei. In table 1 the correlation coefficients $\rho\left(\mathbf{q}, \ln E_{0}\right)$ and $\rho(\mathbf{q}, \ln A)$ between shower parameters $N_{\mathrm{ch}}, N_{\mu}, s$ and primary energy $\left(E_{0}\right)$ and mass of primary nuclei $(A \equiv 1,4,16,56)$ are presented.

We obtained the parametric representation for the energy estimator $\ln E_{1} \simeq f\left(\mathbf{a} \mid N_{\mathrm{ch}}\right.$, $\left.N_{\mu}, s, \theta\right)$ by minimizing $\chi^{2}$,

$$
\chi^{2}=\sum_{A} \sum_{i=1}^{\mathcal{N}} \frac{\left(\ln E_{0, A, i}-\ln E_{1, i}\right)^{2}}{\sigma^{2}}
$$

6 


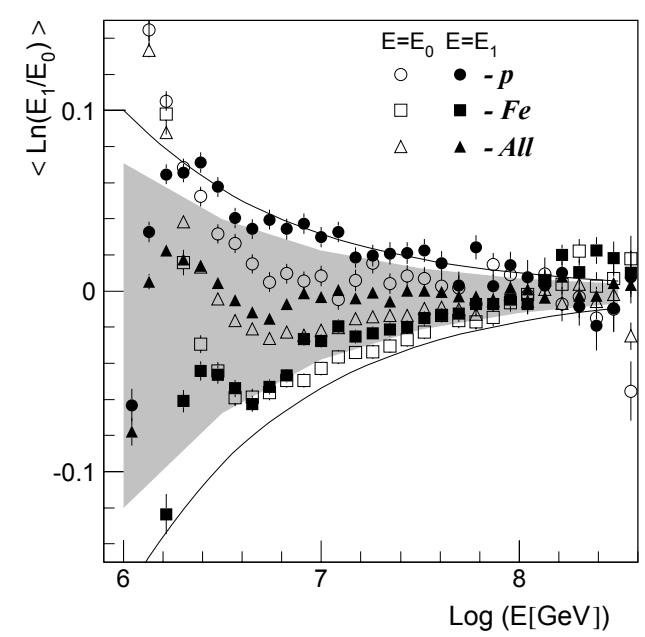

Figure 3. Mean biases versus energies $E \equiv E_{0}$ and $E \equiv E_{1}$ of the primary proton $(p)$ and iron $(\mathrm{Fe})$ nuclei and the uniformly mixed $\mathrm{p}, \mathrm{He}, \mathrm{O}, \mathrm{Fe}$ compositions $(A l l)$.

Table 2. Approximation parameters $a_{1}, \ldots, a_{7}$ of primary energy evaluation (10) obtained from $\chi^{2}$-minimization (9) for the SIBYLL interaction model, $\sigma=0.14$ and $\chi_{\min }^{2} / n_{\text {d.f. }} \simeq 1$.

\begin{tabular}{lllllll}
\hline$a_{1}$ & $a_{2}$ & $a_{3}$ & $a_{4}$ & $a_{5}$ & $a_{6}$ & $a_{7}$ \\
\hline 1.030 & 3.641 & -5.743 & 2.113 & 6.444 & 1.200 & -0.045 \\
\hline
\end{tabular}

with respect to $\mathbf{a} \equiv a\left(a_{1}, a_{2}, \ldots, a_{p}\right)$ for different empirical functions $f\left(\mathbf{a} \mid N_{\mathrm{ch}}, N_{\mu}, s, \theta\right)$ with a different number $(p)$ of unknown parameters. The values of $A, E_{0}$ and the corresponding reconstructed shower parameters $N_{\mathrm{ch}}, N_{\mu}, s$ and $\theta$ for the estimation of $E_{1}$ were taken from the simulated EAS database (section 3.3).

The best energy estimations as a result of the minimization (9) were achieved for the 7-parametric $(p=7)$ fit:

$$
\ln E_{1}=a_{1} x+\frac{a_{2} \sqrt{s}}{c}+a_{3}+a_{4} c+\frac{a_{5}}{\left(x-a_{6} y\right)}+a_{7} y e^{s},
$$

where $x=\ln N_{\mathrm{ch}}, y=\ln N_{\mu}(R<50 \mathrm{~m}), c=\cos \theta, s$ is the shower age and energy $E_{1}$ is in $\mathrm{GeV}$. The values of $a_{1}, \ldots, a_{7}$ parameters are shown in table 2 and were derived at $\sigma=0.14$ and $\chi_{\min }^{2} / n_{\text {d.f. }} \simeq 1$, where the number of degrees of freedom $n_{\text {d.f. }}=8 \times 10^{4}$. The expected errors $\Delta a_{1}, \ldots, \Delta a_{7}$ of corresponding parameters were negligibly small $(\leqslant 5 \%)$ due to very high values of $n_{\text {d.f. }}$.

The corresponding average biases versus energies $\left(E \equiv E_{0}\right.$ and $\left.E \equiv E_{1}\right)$ of the primary proton $(p)$, iron $(F e)$ nuclei and uniformly mixed $\mathrm{p}, \mathrm{He}, \mathrm{O}, \mathrm{Fe}$ composition are presented in figure 3 (symbols). The boundary lines correspond to approximations $\Delta \delta \simeq b / \sqrt{E / 10^{6} \mathrm{GeV}}$, where $b \simeq 0.10$ and $b \simeq-0.17$ for the upper and lower limits respectively. The shaded area corresponds to $b \simeq 0.09$ and $b \simeq-0.15$ and were used to estimate the errors according to (7) for the reconstruction of the all-particle energy spectrum (section 4$)$.

The dependence of standard deviations $\sigma\left(E_{0}\right)$ of systematic errors of energy evaluations (10) on primary energy $E_{0}$ is presented in figure 4 for four primary nuclei and uniformly mixed composition with equal fractions of $\mathrm{p}, \mathrm{He}, \mathrm{O}$ and $\mathrm{Fe}$ nuclei. The results 


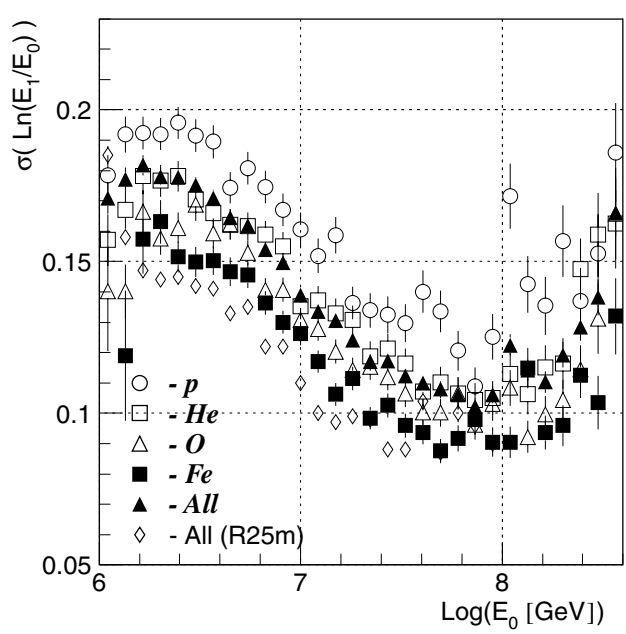

Figure 4. Errors of the energy estimator (6) versus primary energy $E_{0}$ for four primary nuclei and uniformly mixed $(A l l)$ compositions. The empty rhombic symbols are taken from our previous data [10] computed for the mixed composition and shower core selection criteria $R<25 \mathrm{~m}$.

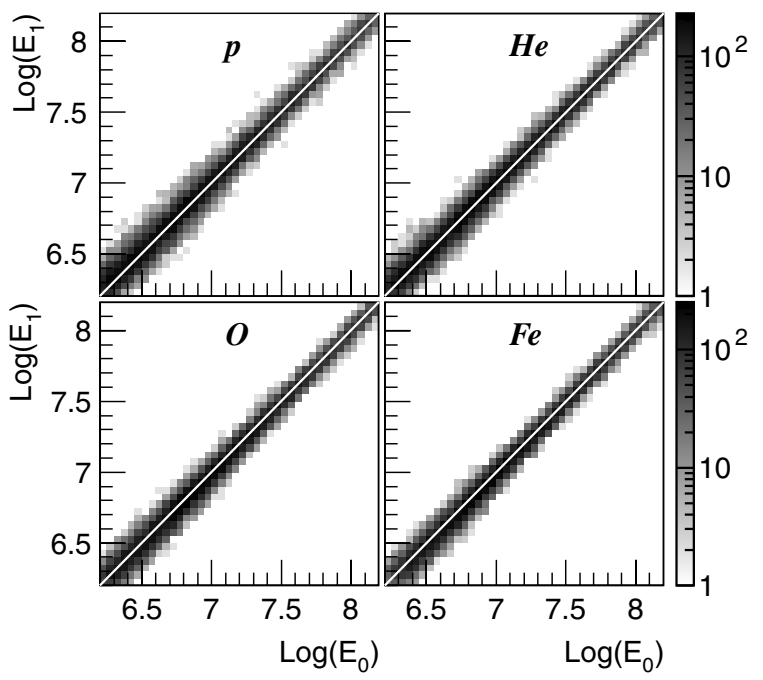

Figure 5. $E_{0}-E_{1}$ (in units of $\mathrm{GeV}$ ) scatter plots for four (p, $\mathrm{He}, \mathrm{O}, \mathrm{Fe}$ ) primary nuclei. The white lines show the corresponding $E_{0}=E_{1}$ dependence.

for the uniformly mixed composition with the shower core selection of $R<25 \mathrm{~m}$ [10] are also presented in figure 4. It is seen that the value of $\sigma=0.14$ responsible for $\chi^{2} \simeq 1$ (expression (9)) with uncertainty $\Delta \sigma \simeq 0.03$ (expression (5)) encloses the $\sigma_{A}\left(E_{0}\right)$ data presented in figure 4.

Such high accuracies of the energy evaluation regardless of primary nuclei are a consequence of the high mountain location of the GAMMA facility $\left(700 \mathrm{~g} \mathrm{~cm}^{-2}\right)$, where the correlation of primary energy with the detected EAS size is about 0.95-0.99 (table 1).

The $E_{0}-E_{1}$ scatter plot of simulated primary energy $E_{0}$ and estimated energy $E_{1}\left(N_{\mathrm{ch}}, N_{\mu}, s, \theta\right)$ according to expression (10) and table 2 is shown in figure 5 . The corresponding distributions of energy errors or the kernel function $W_{A}\left(E_{0}, E_{1} \mid \delta_{A}, \sigma_{A}\right)$ of 


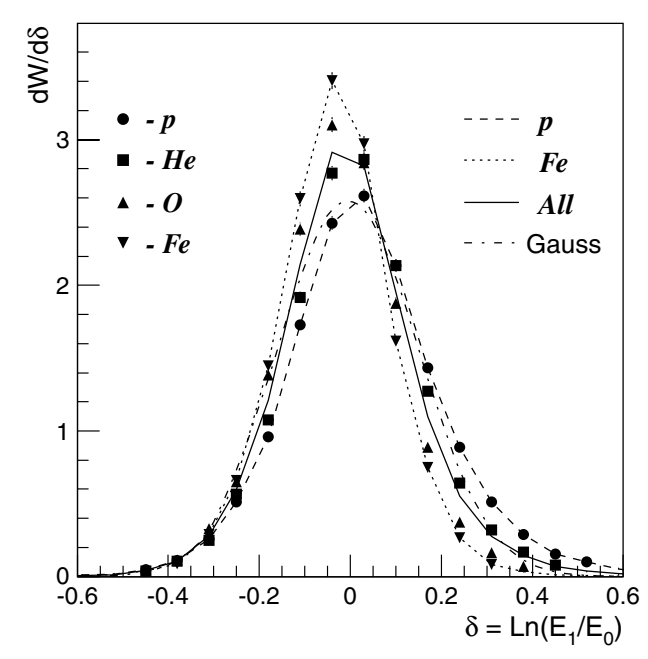

Figure 6. Distribution functions of errors for different primary nuclei (symbols) and uniformly mixed compositions (symbols and solid line).

integral equation (1) for different primary nuclei and uniformly mixed compositions are presented in figure 6 (symbols). The average values $\delta_{A}\left(E_{0}\right)$ and standard deviations $\sigma_{A}\left(E_{0}\right)$ of these distributions depending on energy of the primary nucleus $(A)$ are presented in figures 3 and 4 . The dashed line is an example of the log-normal distribution with $\delta$ and $\sigma$ parameters corresponding to the uniformly mixed composition.

It is seen that the errors can be described by the log-normal distributions and the key assumptions (3)-(5) are approximately valid.

The test of applied approaches (expressions (3)-(6), section 3.2) for the reconstruction of the all-particle primary energy spectrum was carried out by the direct folding of the power-law energy spectrum $\Im\left(E_{0}\right) \equiv \mathrm{d} F_{0} / \mathrm{d} E_{0}$ (expression (8)) with the log-normal kernel function $W\left(E_{0}, E_{1} \mid \delta\left(E_{0}\right), \sigma\left(E_{0}\right)\right)$ according to expression (1) for primary proton and iron nucleus. The values of $\delta\left(E_{0}\right)$ and $\sigma\left(E_{0}\right)$ were derived from the log-parabolic interpolations of corresponding dependences presented in figures 3 and 4 . The event-by-event reconstructed energy spectrum $\mathrm{d} F_{1} / \mathrm{d} E_{1}$ (the left-hand side of expression (1)) was obtained from expression (6) using approaches (3)-(5) with $\sigma=0.14$ and $\Delta \sigma=0.03$. The boundary lines of the shaded area in figure 3 were used as estimations of uncertainties $\Delta \delta\left(E_{1}\right)$ of condition (4). In figure 7 the values of $\left(\mathrm{d} F_{1} / \mathrm{d} E_{1}\right) /\left(\mathrm{d} F_{0} / \mathrm{d} E_{0}\right)$ are presented (symbols) for primary proton and iron nuclei, and different 'unknown' spectral indices of primary energy spectra (8) with the rigidity dependent knee at $E_{k}=3 \times 10^{6} \mathrm{GeV}$. The shaded area is the expected errors computed according to expression (7). It is seen that all spectral discrepancies are practically covered by the expected errors according to expression (7). The star symbols in figure 7 represent the discrepancies in singular spectra with knee at energy $7.4 \times 10^{7} \mathrm{GeV}$ described in section 5 .

\section{All-particle primary energy spectrum}

The EAS dataset analyzed in this paper has been obtained for $5.63 \times 10^{7} \mathrm{~s}$ of live runtime of the GAMMA facility, from 2004 to 2006. Showers to be analyzed were selected with the following criteria: $N_{\mathrm{ch}}>5 \times 10^{5}, R<50 \mathrm{~m}, \theta<45^{\circ}, 0.3<s<1.6, \chi^{2}\left(N_{\mathrm{ch}}\right) / m<3$ and $\chi^{2}\left(N_{\mu}\right) / m<3$ (where $m$ is the number of scintillators with non-zero signal), yielding a 


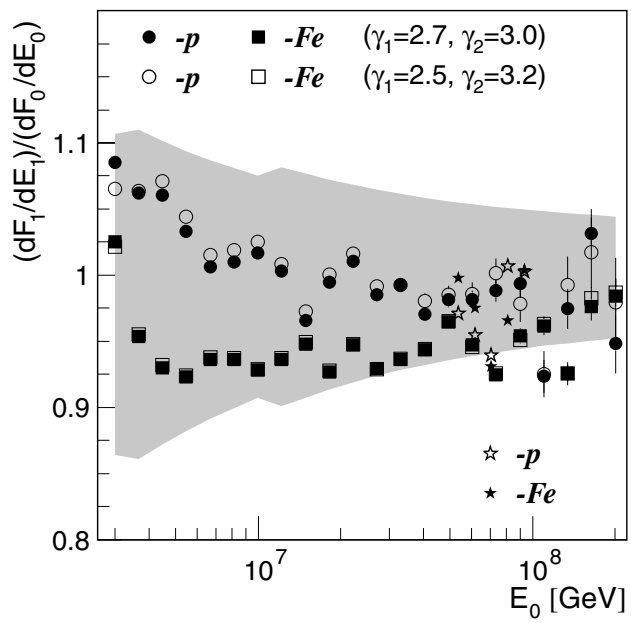

Figure 7. Discrepancies of initial $\left(\mathrm{d} F_{0} / \mathrm{d} E_{0}\right)$ and reconstructed $\left(\mathrm{d} F_{1} / \mathrm{d} E_{1}\right)$ energy spectra (symbols) for the different primary nuclei and spectral indices of initial spectrum. The shaded area shows the expected errors according to expression (7). The star symbols are the spectral discrepancies for a pulsar component (Section 5).

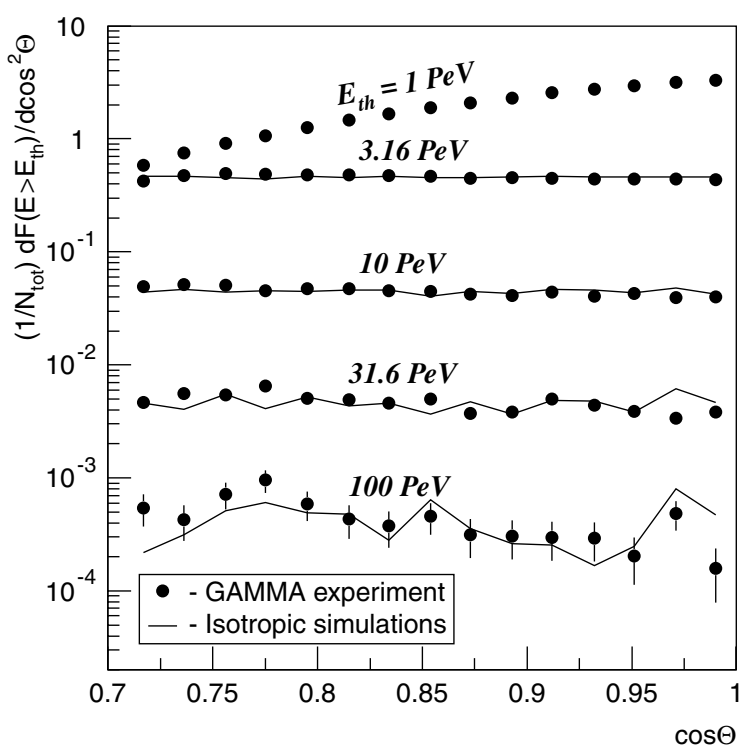

Figure 8. Detected zenith angular distributions for different energy thresholds (symbols). The lines correspond simulated isotropic distributions with the same statistics.

total dataset of $\sim 7 \times 10^{5}$ selected showers. The selected measurement range provided $100 \%$ EAS detection efficiency and similar conditions for the reconstruction of showers produced by primary nuclei $\mathrm{H}, \mathrm{He}, \ldots$, Fe with energies $3 \times 10^{6}<E<(2-3) \times 10^{8} \mathrm{GeV}$. The upper energy limit is determined from figure 4 , where the saturation of surface scintillators in the shower core region begins to be significant.

The independent test of energy estimates can be done by the detected zenith angle distributions which have to be isotropic for different energy thresholds. In figure 8 the 


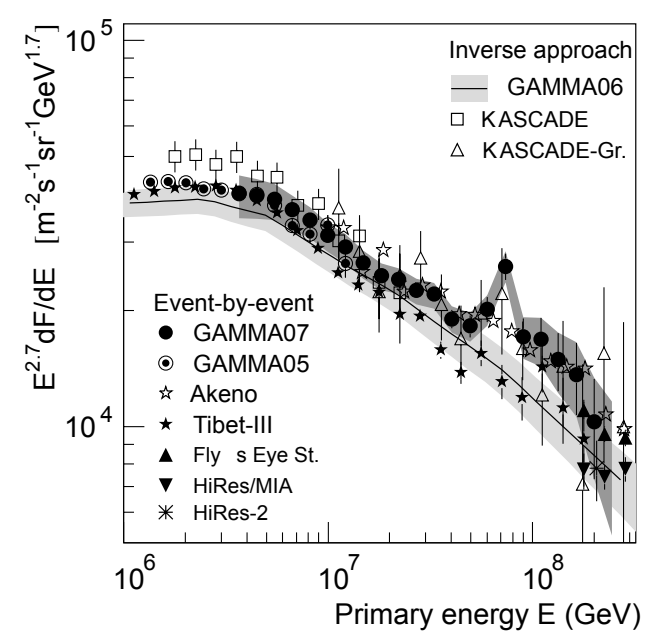

Figure 9. All-particle energy spectrum in comparison with the results of EAS inverse approach $[6,9]$ and our preliminary data [10]. The AKENO, Tibet-III, Fly's Eye Stereo, Hires/MIA and Hires-2 data were taken from [2, 27-30] respectively.

corresponding detected distributions (symbols) are compared with statistically equivalent simulated isotropic distributions (lines). The agreement of detected and simulated distributions at $E>3 \times 10^{6} \mathrm{GeV}$ gives an additional support to the consistency of energy estimates in the whole measurement range. The anisotropic spectral behavior at low energies $(E \sim(1-3) \times$ $10^{6} \mathrm{GeV}$ ) is explained by the lack of heavy nuclei at larger zenith angles in the detected flux due to the applied shower selection criteria.

Using the aforementioned unbiased $(<5 \%)$ event-by-event method of primary energy evaluation (10), we obtained the all-particle energy spectrum. Results are presented in figure 9 (filled circle symbols, GAMMA07) in comparison with the same spectra obtained by the EAS inverse approach (line with shaded area, GAMMA06) from [6,9] and our preliminary results (point-circle symbols, GAMMA05) obtained using the 7-parametric event-by-event method with the shower core selection criteria $R<25 \mathrm{~m}$ and $\theta<30^{\circ}$ [10].

It follows from our preliminary data $[10,11]$ that the all-particle energy spectrum derived using event-by-event analysis with the multi-parametric energy estimator (section 3) depends only slightly on the interaction model (QGSJET01 [25] or SIBYLL2.1 [14]) and thereby, the errors of obtained spectra are mainly determined by the sum of statistical and systematic errors (7) presented in figure 9 by the dark shaded area.

The shower size detection threshold effects distort the all-particle spectrum in the range of $E<(2-2.5) \times 10^{6} \mathrm{GeV}$ depending on the interaction model and determine the lower limit $E_{\min }=3 \times 10^{6} \mathrm{GeV}$ of the energy spectrum in figure 9 whereas the upper limit of the spectrum $E_{\max } \simeq(2-3) \times 10^{8} \mathrm{GeV}$ is determined by the saturation of our shower detectors which begins to be significant at $E_{\mathrm{p}}>2 \times 10^{8} \mathrm{GeV}$ and $E_{\mathrm{Fe}}>4 \times 10^{8} \mathrm{GeV}$ (see figure 4) for primary proton and Fe nuclei. The range of minimal systematic errors and biases is $(1-10) \times 10^{7} \mathrm{GeV}$, where about $13 \%$ and $10 \%$ errors were attained (figures 3,4 ) for primary $\mathrm{H}$ and $\mathrm{Fe}$ nuclei respectively.

In table 3 the numerical values of the obtained all-particle energy spectrum are presented along with statistical, total upper and lower errors according to (7) and corresponding number of detected events. The energy spectra for the low energy region (the first four lines) were taken from our data [10] for the EAS selection criteria $R<25 \mathrm{~m}$ and $\theta<30^{\circ}$. 
Table 3. All-particle energy spectrum $(\mathrm{d} \Im / \mathrm{d} E)$ in units of $\left(\mathrm{m}^{2} \mathrm{sec} \mathrm{sr} \mathrm{GeV}\right)^{-1}$ and corresponding statistical $\left(\Delta_{\text {stat }}\right)$, total upper, $\left(\Delta_{+}\right)$and total lower $\left(\Delta_{-}\right)$errors and number of events $\left(N_{\mathrm{ev}}\right)$. The first four lines represent our data [10] obtained for selection criteria $R<25 \mathrm{~m}$ and $\theta<30^{\circ}$.

\begin{tabular}{|c|c|c|c|c|c|}
\hline$E(P e V)$ & $\mathrm{d} \Im / \mathrm{d} E$ & $\Delta_{\text {stat }}$ & $\Delta_{+}$ & $\Delta_{-}$ & $N_{\mathrm{ev}}$ \\
\hline $1.35[10]$ & $0.1205 \times 10^{-11}$ & $0.11 \times 10^{-13}$ & - & - & 11550 \\
\hline $1.65[10]$ & $0.7037 \times 10^{-12}$ & $0.77 \times 10^{-14}$ & - & - & 8374 \\
\hline $2.01[10]$ & $0.4090 \times 10^{-12}$ & $0.54 \times 10^{-14}$ & - & - & 5769 \\
\hline $2.46[10]$ & $0.2285 \times 10^{-12}$ & $0.36 \times 10^{-14}$ & - & - & 3924 \\
\hline 3.00 & $0.1297 \times 10^{-12}$ & $0.52 \times 10^{-15}$ & $0.16 \times 10^{-13}$ & $0.20 \times 10^{-13}$ & 59930 \\
\hline 3.67 & $0.7677 \times 10^{-13}$ & $0.37 \times 10^{-15}$ & $0.86 \times 10^{-14}$ & $0.108 \times 10^{-13}$ & 43157 \\
\hline 4.48 & $0.4401 \times 10^{-13}$ & $0.25 \times 10^{-15}$ & $0.45 \times 10^{-14}$ & $0.57 \times 10^{-14}$ & 30153 \\
\hline 5.47 & $0.2524 \times 10^{-13}$ & $0.17 \times 10^{-15}$ & $0.24 \times 10^{-14}$ & $0.30 \times 10^{-14}$ & 20993 \\
\hline 6.69 & $0.1372 \times 10^{-13}$ & $0.12 \times 10^{-15}$ & $0.12 \times 10^{-14}$ & $0.15 \times 10^{-14}$ & 13917 \\
\hline 8.17 & $0.7506 \times 10^{-14}$ & $0.77 \times 10^{-16}$ & $0.62 \times 10^{-15}$ & $0.76 \times 10^{-15}$ & 9290 \\
\hline 9.97 & $0.3984 \times 10^{-14}$ & $0.51 \times 10^{-16}$ & $0.31 \times 10^{-15}$ & $0.37 \times 10^{-15}$ & 5998 \\
\hline 12.18 & $0.2166 \times 10^{-14}$ & $0.34 \times 10^{-16}$ & $0.17 \times 10^{-15}$ & $0.21 \times 10^{-15}$ & 3986 \\
\hline 14.88 & $0.1148 \times 10^{-14}$ & $0.23 \times 10^{-16}$ & $0.87 \times 10^{-16}$ & $0.104 \times 10^{-15}$ & 2573 \\
\hline 18.17 & $0.619 \times 10^{-15}$ & $0.15 \times 10^{-16}$ & $0.45 \times 10^{-16}$ & $0.53 \times 10^{-16}$ & 1692 \\
\hline 22.20 & $0.350 \times 10^{-15}$ & $0.10 \times 10^{-16}$ & $0.25 \times 10^{-16}$ & $0.29 \times 10^{-16}$ & 1170 \\
\hline 27.11 & $0.1927 \times 10^{-15}$ & $0.69 \times 10^{-17}$ & $0.13 \times 10^{-16}$ & $0.15 \times 10^{-16}$ & 781 \\
\hline 33.12 & $0.1101 \times 10^{-15}$ & $0.47 \times 10^{-17}$ & $0.78 \times 10^{-17}$ & $0.88 \times 10^{-17}$ & 542 \\
\hline 40.45 & $0.556 \times 10^{-16}$ & $0.30 \times 10^{-17}$ & $0.42 \times 10^{-17}$ & $0.46 \times 10^{-17}$ & 334 \\
\hline 49.40 & $0.306 \times 10^{-16}$ & $0.20 \times 10^{-17}$ & $0.26 \times 10^{-17}$ & $0.27 \times 10^{-17}$ & 227 \\
\hline 60.34 & $0.199 \times 10^{-16}$ & $0.15 \times 10^{-17}$ & $0.18 \times 10^{-17}$ & $0.19 \times 10^{-17}$ & 178 \\
\hline 73.70 & $0.149 \times 10^{-16}$ & $0.12 \times 10^{-17}$ & $0.13 \times 10^{-17}$ & $0.14 \times 10^{-17}$ & 164 \\
\hline 90.02 & $0.572 \times 10^{-17}$ & $0.65 \times 10^{-18}$ & $0.70 \times 10^{-18}$ & $0.71 \times 10^{-18}$ & 77 \\
\hline 110.0 & $0.326 \times 10^{-17}$ & $0.44 \times 10^{-18}$ & $0.47 \times 10^{-18}$ & $0.47 \times 10^{-18}$ & 54 \\
\hline 134.3 & $0.184 \times 10^{-17}$ & $0.30 \times 10^{-18}$ & $0.31 \times 10^{-18}$ & $0.31 \times 10^{-18}$ & 34 \\
\hline 164.0 & $0.94 \times 10^{-18}$ & $0.19 \times 10^{-18}$ & $0.20 \times 10^{-18}$ & $0.20 \times 10^{-18}$ & 22 \\
\hline 200.3 & $0.40 \times 10^{-18}$ & $0.11 \times 10^{-18}$ & $0.12 \times 10^{-18}$ & $0.12 \times 10^{-18}$ & 12 \\
\hline 244.7 & $0.243 \times 10^{-18}$ & $0.81 \times 10^{-19}$ & $0.82 \times 10^{-19}$ & $0.82 \times 10^{-19}$ & 7 \\
\hline
\end{tabular}

The obtained energy spectrum agrees within errors with the KASCADE [6], AKENO [2] and Tibet-III [27] data both in the slope and in the absolute intensity practically in the whole measurement range. Looking at the experimental points we can unambiguously point out the existence of an irregularity in the spectrum at the energy of (6-8) $\times 10^{7} \mathrm{GeV}$. As is seen from figures 3 and 4 , the energy estimator (10) has minimal biases $(\sim 4-5 \%)$ and errors $(\sim 0.09-0.12)$ at this energy. With these errors the obtained bump has an apparently real nature. If we fit all our other points in the $(5-200) \times 10^{6} \mathrm{GeV}$ energy range by a smooth power-law spectrum, the bin at $7.4 \times 10^{7} \mathrm{GeV}$ exceeds this smooth spectrum by 4.0 standard deviations. The exact value for this significance of the bump depends somewhat on the energy range chosen to adjust the reference straight line in figure 9, but it lies in the range (3.5-4.5) $\sigma$.

We conservatively included the systematic errors in this estimate, although they are not independent at the nearby points but correlated: the possible overestimation of the energy at one point cannot be followed by an underestimation at the neighboring point if their energies are relatively close to each other. Systematic errors can change slightly the general slope of the spectrum but cannot imitate the fine structure and the existence of the bump.

The results from figure 7 show that in the range of 'bump' energy $\left(7.4 \times 10^{7} \mathrm{GeV}\right)$ the systematic errors cannot significantly increase the flux. To test this hypothesis more precisely we tested the reconstruction procedure for singular energy spectra with power indices $\gamma_{\mathrm{p}}=1.5$ 
and $\gamma_{\mathrm{p}}=4.5$ before and above the knee energy $7.4 \times 10^{7} \mathrm{GeV}$ in the $5 \times 10^{7}-10^{8} \mathrm{GeV}$ energy range. Results are presented in figure 7 (star symbols) and show that there are no significant discrepancies in the reconstructed spectra observed, which stems from high accuracy of energy reconstruction.

The detected shower sample in the bump energy region did not reveal any discrepancies in the showers from adjacent energy bins within statistical errors neither with respect to reconstructed shower core coordinates, zenith angular and $\chi^{2}$ distributions, nor with respect to $\xi=N_{\mathrm{ch}} / N_{\mu}$ distribution. The only difference is that the average age of showers increases from $\bar{s}=0.88 \pm 0.007$ at $E_{0} \simeq 5 \times 10^{7} \mathrm{GeV}$ up to $\bar{s}=0.93 \pm 0.01$ at $E_{0} \simeq 10^{8} \mathrm{GeV}$, instead of the monotonous shower age decrease with energy increment, which is observed at a low-energy region $\left(E_{0}=3 \times 10^{6}-5 \times 10^{7} \mathrm{GeV}\right)$.

It is necessary to note that some indications of the observed bump are also seen in KASCADE-Grande [6] (figure 9), TUNKA [4] and Tibet-III [27] data but with larger statistical uncertainties at the level of 1.5-2 standard deviations. Moreover, the locations of the bump in different experiments agree well with each other and with an expected knee energy for Fe-like primary nuclei according to the rigidity-dependent knee hypothesis [8, 9]. However, the observed width ( $\sim 20 \%$ in energy) and height of the bump at the energy of $(6-8) \times$ $10^{7} \mathrm{GeV}$, which exceeds by a factor of $\sim 1.5(\sim 4$ standard deviations) the best fit straight line fitting all points above $5 \times 10^{6} \mathrm{GeV}$ in figure 9, are difficult to describe in the framework of the conventional model of cosmic ray origin [21].

As will be shown below (section 5, figures 10,11) the detected EAS charged particle $\left(N_{\mathrm{ch}}\right)$ and muon size $\left(N_{\mu}\right)$ spectra $[8,9]$ independently indicate the existence of this bump for the obtained energies and as this follows from the behavior of the shower age parameter versus shower size $[8,9]$, the bump at energy $\sim 7.4 \times 10^{7} \mathrm{GeV}$ is likely formed completely from Fe nuclei.

\section{Possible origin of irregularities}

Irregularities of the all-particle energy spectrum in the knee region are observed practically in all measurements $[2,6,8]$ and are explained by both the rigidity-dependent knee hypothesis and contribution of pulsars in the Galactic cosmic ray flux [20, 31, 32]. Two of these approaches approximately describe the all-particle spectrum in the (1-100) $\times 10^{6} \mathrm{GeV}$ energy region. However, the observed bump in figure 9 at energies $\sim 7.4 \times 10^{7} \mathrm{GeV}$ both directly points out the presence of additional component in the primary nuclei flux and displays a very flat $\left(\gamma_{\mathrm{p}} \sim 0-2\right)$ energy spectrum before a cut-off energy of $E_{\mathrm{c}} \simeq 8 \times 10^{7} \mathrm{GeV}$.

It is known [8, 9] that rigidity-dependent primary energy spectra cannot describe quantitatively the phenomenon of ageing of EAS at energies $(5-10) \times 10^{7} \mathrm{GeV}$ which was observed in the most mountain-altitude experiments [8, 15, 33]. It is reasonable to assume that an additional flux of heavy nuclei (Fe-like) is responsible for the bump at these energies. Besides, the sharpness of the bump (figure 9) points out the local origin of this flux from compact objects (pulsars) [31, 32].

We carried out the test of this hypothesis using the parameterized inverse approach [7-9] on the basis of the GAMMA facility EAS database and the hypothesis of two-component origin of cosmic ray flux:

$$
F_{A}(E)=\Phi_{G}(A)\left(E_{k}^{-\gamma_{1}}\left(\frac{E}{E_{k}}\right)^{-\gamma}+P_{A}(E)\right)
$$




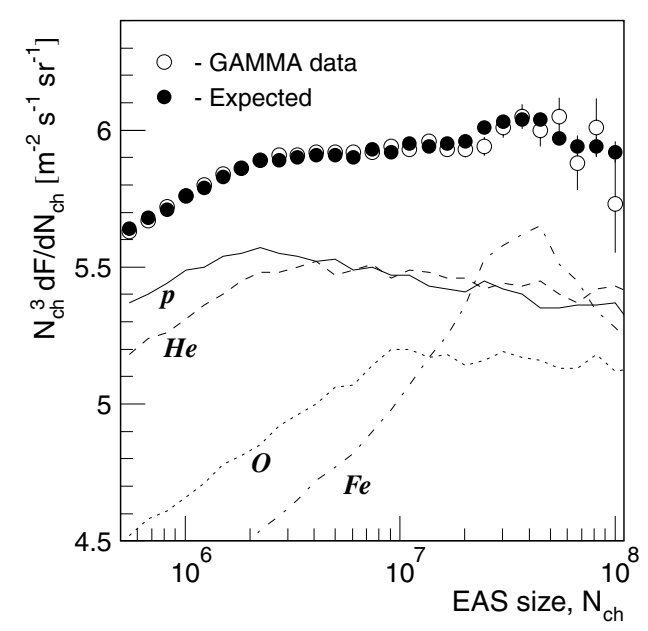

Figure 10. EAS size spectra detected by the GAMMA facility (empty symbols) and corresponding expected spectra (filled symbols) computed in the framework of the SIBYLL2.1 interaction model and the two-component parametrization of primary spectra (11). The lines correspond to expected size spectra computed for each of the primary nuclei.

where $P_{\mathrm{H}}=P_{\mathrm{He}}=P_{\mathrm{O}}=0$ and

$$
P_{\mathrm{Fe}}(E)=\Phi_{P}(\mathrm{Fe}) \cdot E_{\mathrm{c}, \mathrm{Fe}}^{-\gamma_{1 \mathrm{p}}}\left(\frac{E}{E_{\mathrm{c}, \mathrm{Fe}}}\right)^{-\gamma_{\mathrm{p}}} .
$$

The first term on the right-hand side of expression (11) (the so-called Galactic component) is the power-law energy spectra with rigidity-dependent knees at energies $E_{k}=E_{R} \cdot Z$ and power indices $\gamma=\gamma_{1}$ and $\gamma=\gamma_{2}$ for $E \leqslant E_{k}$ and $E>E_{k}$ respectively, and the second term (the so-called pulsar component) is an additional power-law energy spectrum with cut-off energies $E_{\mathrm{c}, \mathrm{Fe}}$ and power indices $\gamma_{\mathrm{p}}=\gamma_{1, \mathrm{p}}$ and $\gamma_{\mathrm{p}}=\gamma_{2 \mathrm{p}}$ for $E \leqslant E_{\mathrm{c}, \mathrm{Fe}}$ and $E>E_{\mathrm{c}, \mathrm{Fe}}$ respectively.

The scale factors $\Phi_{G}(A)$ and $\Phi_{P}(A)$ along with particle rigidity $E_{R}$, cut-off energy $E_{\mathrm{c}}(A)$ and power indices $\gamma_{1}, \gamma_{2}, \gamma_{1 \mathrm{p}}, \gamma_{2 \mathrm{p}}$ were estimated using the combined approximation method [7-9] for two examined shower spectra shown in figures 10 and 11 (empty symbols): EAS size spectra, $\mathrm{d} F / \mathrm{d} N_{\text {ch }}$ (figure 10) and EAS muon truncated size spectra, $\mathrm{d} F / \mathrm{d} N_{\mu}$ (figure 11) detected by the GAMMA facility using the shower core selection criteria $\theta<30^{\circ}$ and $r<$ $50 \mathrm{~m}[8,9]$. We did not consider the $\mathrm{H}, \mathrm{He}$ and $\mathrm{O}$ pulsar components to avoid a large number of unknown parameters and the corresponding mutually compensative pseudo solutions [34] for the galactic components.

The folded (expected) shower spectra (filled symbols in figures 10,11) were computed on the basis of parametrization (11) and CORSIKA EAS simulated data set $[8,9]$ for the $A \equiv$ $\mathrm{H}, \mathrm{He}, \mathrm{O}$ and $\mathrm{Fe}$ primary nuclei to evaluate the kernel functions of corresponding integral equations $[8,9]$. The computation method was completely the same as was performed in the combined approximation analysis $[8,9]$. The initial values of spectral parameters for the galactic component were taken from [8,9] as well. In figures 10 and 11 we also presented the derived expected elemental shower spectra (lines) for primary $\mathrm{H}, \mathrm{He}, \mathrm{O}$ and $\mathrm{Fe}$ nuclei, respectively.

The parameters of two-component primary energy spectra (11) derived from the $\chi^{2}$ goodness-of-fit test of shower spectra $\mathrm{d} F / \mathrm{d} N_{\text {ch }}$ and $\mathrm{d} F / \mathrm{d} N_{\mu}$ are presented in table 4. 


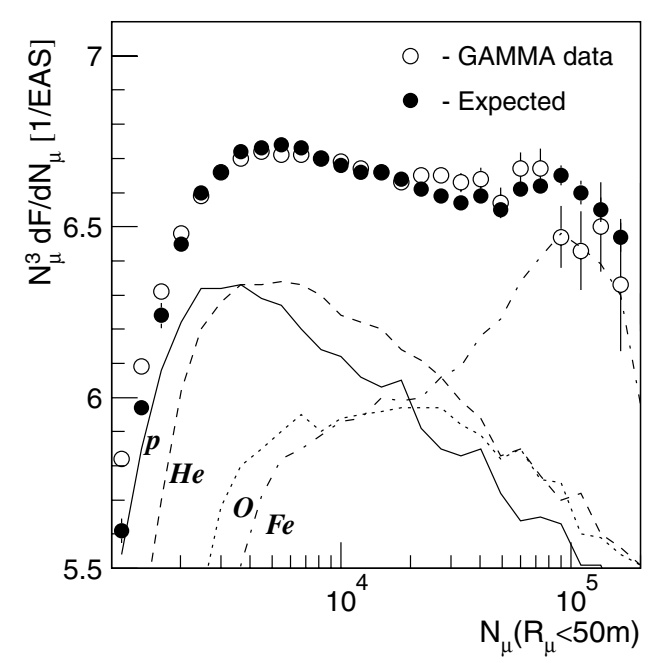

Figure 11. Same as figure 10 for truncated EAS muon size spectra.

Table 4. Parameters of the primary energy spectra (11) derived from combined approximations of detected shower spectra. The scale factors $\Phi_{G, P}(A)$ have units of $\left(\mathrm{m}^{2} \mathrm{~s} \mathrm{sr} \mathrm{TeV}\right)^{-1}$. The particle rigidity $E_{R}$ and cut-off energies $E_{\mathrm{c}}$ are shown in $\mathrm{PV}$ and $\mathrm{PeV}$ units respectively.

\begin{tabular}{lll}
\hline Param. & G-component & P-component \\
\hline$\Phi(\mathrm{H})$ & $0.102 \pm 0.003$ & - \\
$\Phi(\mathrm{He})$ & $0.094 \pm 0.022$ & - \\
$\Phi(\mathrm{O})$ & $0.032 \pm 0.015$ & - \\
$\Phi(\mathrm{Fe})$ & $0.021 \pm 0.006$ & $(0.29 \pm 0.08) \times 10^{-7}$ \\
$\gamma_{1}$ & $2.68 \pm 0.005$ & $1.05 \pm 0.5$ \\
$\gamma_{2}$ & $3.29 \pm 0.045$ & $4.5 \pm 0.4$ \\
$E_{\mathrm{R}}$ & $2.59 \pm 0.15$ & - \\
$E_{\mathrm{c}, \mathrm{Fe}}$ & - & $76.9 \pm 1.5$ \\
\hline
\end{tabular}

Resulting expected energy spectra $F_{A}(E)$ for the Galactic $\mathrm{H}, \mathrm{He}, \mathrm{O}$ and $\mathrm{Fe}$ nuclei (thin lines) along with the all-particle spectrum $\sum_{A} F_{A}(E)$ (bold line with shaded area) are presented in figure 12. The thick dash-dotted line corresponds to derived energy spectra of the additional Fe component (the second term on the right-hand side of expression (11)). The all-particle energy spectrum obtained on the basis of the GAMMA EAS data and the event-by-event multiparametric energy evaluation method (section 4, figure 9) is shown in figure 12 (symbols) as well.

It is seen that the shape of the two-component all-particle spectrum (bold line with shaded area) calculated with parameters taken from the fit of EAS size spectra agrees within the errors with the results of the event-by-event analysis (symbols) that points out the consistency of applied spectral parametrization (11) with GAMMA data.

Note that the flux of the derived additional Fe component turned out to be about $0.5-0.6 \%$ of the total Fe flux for primary energies $E>10^{6} \mathrm{GeV}$. This result agrees with the expected flux of polar cap component [20].

The dependence of average nuclear mass number is presented in figure 13 for two primary nuclei flux composition models: one-component model, where the power-law energy spectra of primary nuclei have rigidity-dependent knees at particle rigidity $E_{\mathrm{R}} \sim 2500 \mathrm{GeV} / \mathrm{Z}$ [8, 9] (the so-called Galactic component, dashed line) and two-component model (solid line), where 


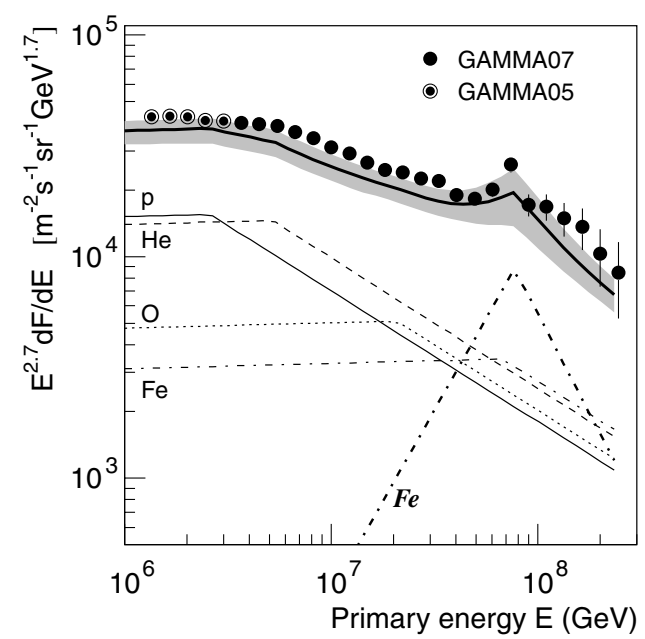

Figure 12. All-particle primary energy spectrum (symbols) and expected energy spectra (lines and shaded area) derived from EAS inverse problem solutions for $\mathrm{p}, \mathrm{He}, \mathrm{O}$ and $\mathrm{Fe}$ primary nuclei using the two-component parametrization (11). The thin lines are the energy spectra of Galactic $\mathrm{H}, \mathrm{He}$, $\mathrm{O}$ and $\mathrm{Fe}$ components. The thick dash-dotted line is an additional Fe component from compact objects.

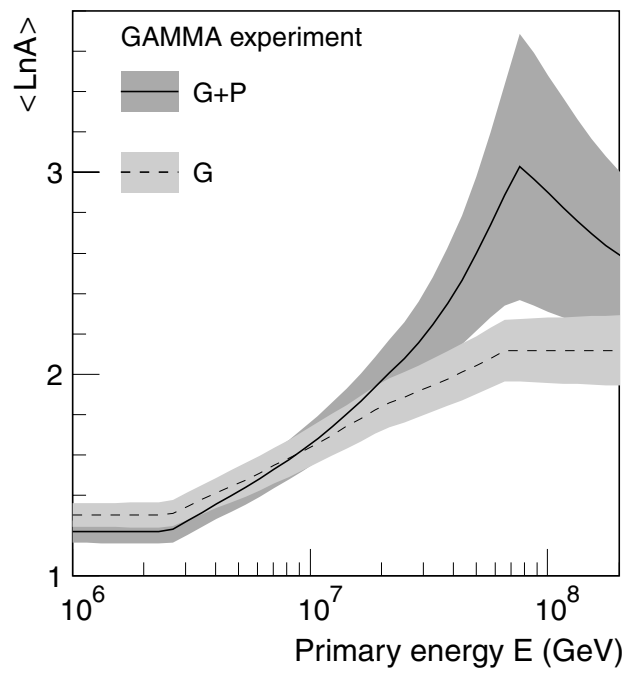

Figure 13. Average logarithm primary nuclei mass number derived from rigidity-dependent primary energy spectra [8,9] (dashed line) and 2-component model prediction (11) taking into account additional pulsar component (solid line).

an additional pulsar $(P)$ component was included according to parametrization (11) and data from table 4 with a very flat power index $\left(\gamma_{1 \mathrm{p}} \sim 1\right)$ before the cut-off energy $E_{\mathrm{c}, \mathrm{Fe}}$. The shaded area in figure 13 shows the ranges of total (systematic and statistical) errors.

\section{Conclusion}

The multi-parametric event-by-event method (sections 3,4) provides high accuracy for the energy evaluation of primary cosmic ray nuclei $\sigma(E) \simeq 10-15 \%$ regardless of the nuclei 
mass (biases $<5 \%$ ) in the 3-200 PeV energy region. Using this method the all-particle energy spectrum in the knee region and above has been obtained (figure 9, table 3) using the EAS database from the GAMMA facility. The results are obtained for the SIBYLL2.1 interaction model.

The all-particle energy spectrum in the range of statistical and systematic errors agrees with the same spectra obtained using the EAS inverse approach [6-8] in the 3-200 PeV energy range.

The high accuracy of energy evaluations and small statistical errors point out at the existence of an irregularity ('bump') in the 60-80 PeV primary energy region.

The bump can be described by a two-component model (parametrization (11)), of primary cosmic ray origin, where additional (pulsar) Fe components are included with very flat powerlaw energy spectrum $\left(\gamma_{1 \mathrm{p}} \sim 1 \pm 0.5\right)$ before the cut-off energy $E_{\mathrm{c}, \mathrm{Fe}}$ (figure 13 , table 4$)$. At the same time, the EAS inverse problem solutions for energy spectra of pulsar component force the solutions for the slopes of Galactic component behind the knee to be steeper (Table 4), which creates a problem of underestimation of the all-particle energy spectrum in the range of HiRes [29] and Fly's Eye [28] data at $E>200 \mathrm{PeV}$. From this viewpoint the underestimation (10-15\%) of the all-particle energy spectrum (bold solid line in figure 12) in the range of $E>200 \mathrm{PeV}$ can be compensated for by the expected extragalactic component [35].

Though we cannot reject the stochastic nature of the bump completely, our examination of the systematic uncertainties of the applied method lets us believe that they cannot be responsible for the observed feature. The indications from other experiments mentioned in this paper provide the argument for the further study of this interesting energy region.

\section{Acknowledgments}

We are grateful to all our colleagues at the Moscow P N Lebedev Physical Institute and the Yerevan Physics Institute who took part in the development and exploitation of the GAMMA array. We thank Peter Biermann for fruitful discussions. This work has been partly supported by the research grant no. 090 from the Armenian government, the RFBR grant 07-02-00491 in Russia, and the 'Hayastan' All-Armenian Fund and the ECO-NET project 12540UF.

\section{References}

[1] Erlykin A D and Wolfendale A W 1997 J. Phys. G: Nucl. Part. Phys. 23979 Erlykin A D and Wolfendale A W 1999 Astron. Astrophys. 350 L1 Erlykin A D and Wolfendale A W 2001 J. Phys. G: Nucl. Part. Phys. 271005

[2] Nagano N et al 1984 J. Phys. G: Nucl. Phys. 101295

[3] Garyaka A P et al 2002 J. Phys. G: Nucl. Part. Phys. 282317

[4] Korosteleva E E et al 2007 Nucl. Phys. B (proc. suppl.) 16574

[5] Antoni T et al 2005 Astropart. Phys. 241 (Preprint astro-ph/0505413)

[6] Bruggemann M et al 2006 Proc. 20th ECRS (Lisbon, 2006) available at http://www.lip.pt/events/2006/ecrs/ proc/ercs06-s3-77.pdf

[7] Ter-Antonyan S V et al 2005 29th ICRC, HE1.2, Pune vol 6 p 101 (Preprint astro-ph/0506588)

[8] Ter-Antonyan S V et al 2007 Proc. Inter. Cosmic Ray Workshop Aragats-2007, (Nor-Amberd, Armenia) p 33, available at http://gamma.yerphi.am/

[9] Garyaka A P et al 2007 Astropart. Phys. 28169 (Preprint arXiv:0704.3200)

[10] Ter-Antonyan S V et al 2005 29th ICRC, HE1.2, Pune vol 6 p105

[11] Ter-Antonyan S V et al 2005 Preprint astro-ph/0506588

[12] Chilingarian A, Ter-Antonian S and Vardanyan A 1997 Nucl. Phys. B 52B 240

[13] Eganov V et al 2005 Int. J. Mod. Phys. A 206811

[14] Fletcher R S, Gaisser T K, Lipari P and Stanev T 1994 Phys. Rev. D 505710

[15] Avakian V V et al 1995 Proc. 24th Int. Cosmic Ray Conf. Rome vol 1 p348 
[16] Murzin V S and Sarycheva L I 1968 Cosmic Rays and Their Interactions (Moscow: Atomizd) (in Russian)

[17] Aglietta M et al (EAS-TOP Collaboration) 1999 Astropart. Phys. 101

[18] Glasmacher M A K et al 1999 Astropart. Phys. 10291

[19] Amenomori M et al 2005 29th ICRC, HE1.2 (Pune): (29icrc/PAPERS/HE12/jap-katayose-Y-abs2-he12poster.pdf)

[20] Stanev T, Biermann P L and Gaisser T K 1993 Astron. Astrophys. 274902

[21] Hillas A M 2005 J. Phys. G: Nucl. Part. Phys. 31 R95

[22] Peters B 1959 Nuovo Cimento (Suppl.) 14436

[23] Hörandel J R 2004 Astropart. Phys. 21241

[24] Heck D, Knapp J, Capdevielle J N, Schatz G and Thouw T 1998 Forschungszentrum Karlsruhe Report FZKA 6019

[25] Kalmykov N N and Ostapchenko S S 1993 Yad. Fiz. 56105 (in Russian)

[26] Fasso A, Ferrari A and Roesler S et al 2003 Preprint hep-ph/0306267 (http://www.fluka.org)

[27] Amenomori M et al 2008 Preprint arXiv:0801.1803

[28] Bird D J et al 1995 Astrophys. J. 441144

[29] Abu-Zayyad T et al 2001 Astrophys. J. 557557

[30] Abbassi R U et al 2002 Preprint astro-ph/0208301

[31] Erlykin A D and Wolfendale A W 2004 Astropart. Phys. 2247 (Preprint astro-ph/0404530)

[32] Bednarek W and Protheroe R J 2002 Astropart. Phys. 16397

[33] Miyake S et al 1979 Proc. 16th ICRC, Kyoto vol 13 p171

[34] Ter-Antonyan S V 2007 Astropart. Phys. 28321

[35] Gaisser T K 2005 Preprint astro-ph/0501195 